\title{
ECG changes in patients on chronic psychotropic medication
}

M Y H Moosa, MMedPsych, FCPsych, MB ChB, MCFP, BSC

F Y Jeenah, MMed, Psych, MB ChB

C Mouton, $\mathrm{MB} \mathrm{ChB}$

Department of Neurosciences, University of the Witwatersrand, Johannesburg

Objectives. To determine the ECG changes in a group of outpatients on chronic psychotropic medication, and the association, if any, with factors such as gender, age, comorbid illness and the use of concomitant medication.

Methods. Study subjects included patients 18 years and older attending the outpatient departments of Chris Hani Baragwanath and Johannesburg hospitals. The subjects' demographic and clinical characteristics were obtained and a resting ECG was recorded.

Results. Eighty patients were included in the study. The mean age of the subjects was 45.4 (standard deviation (SD) $=18.2$ ) years, with a minimum age of 18 and a maximum of 86 years. Fifty-four subjects (67.5\%) had evidence of some ECG abnormalities. There was no significant difference between the occurrence of ECG abnormalities and the different age groups $(p>0.05)$, gender $(p>0.05)$, and different race groups ( $p>0.05)$. Sixty-one subjects (76.3\%) had no co-morbid medical illness and were on psychotropic medication only; of these patients 43 (70.5\%) had abnormal ECG tracings ( $p>$ 0.05). The ECG abnormalities recorded included abnormal rate $(28.8 \%)$, abnormal ST segment $(20.5 \%)$, abnormal QRS complex (17.8\%), abnormal T wave (15.4\%), prolonged or borderline corrected QT interval $(8.2 \%)$, irregular rhythm $(5.5 \%)$ and prolonged PR interval $(2.7 \%)$. There was a significant positive correlation between the corrected QT interval and age $(r=0.43, p<0.05)$ and between corrected QT interval and female gender $(r=0.31, p<0.05)$. There was no correlation between corrected QT interval and treatment of a co-morbid illness $(r=0.13, p>0.05)$.

Conclusion. The use of psychotropic drugs is associated with ECG changes in ordinary doses. However, this study serves to strengthen previous evidence that, although common, most of these changes are of a benign nature.
Psychiatric patients have been identified as a population at risk for cardiovascular problems., ${ }^{1,2}$ Mortality rates are higher among psychiatric patients than in the general population ${ }^{3}$ and pharmaclogical treatment may produce side-effects that affect mortality. ${ }^{4}$ In addition, certain cardiac risk factors (smoking, lack of exercise, obesity, substance misuse) and high autonomic arousal during physical restraint are overrepresented in psychiatric patients. Consequently, in recent years there has been increasing concern about the cardiac safety of psychotropic medication and the safe selection of these drugs.

\section{Cardiac conduction}

Psychotropic drugs with antimuscarinic and anticholinergic effects lall low-potency typical antipsychotics, some atypical antipsychotics, most tricyclic antidepressants, non-selective monoamine oxidase inhibitors and all anti-Parkinsonian anticholinergics) may cause sinus tachycardia. However, sinus tachycardia rarely leads to any symptoms and usually remits over

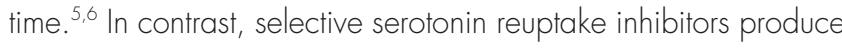
minor degrees of bradycardia. Tricyclic antidepressants also delay cardiac conduction, mainly by prolonging the QRS interval. The effect is dose-dependent, occurring at both therapeutic and toxic doses, particularly with amitriptyline and imipramine. ${ }^{7,8}$ This effect is unlikely to be of clinical relevance in an otherwise normal heart; however, it might precipitate complete heart block in the presence of pre-existing conduction delay such as bundle-branch block or second and higher degrees of heart block.

\section{Cardiac repolarisation}

\section{T-wave changes}

T-wave changes (T-wave broadening, blunting without loss of amplitude, loss of amplitude and bifid T-wave and flattened/ inverted T-wave) usually normalise in the majority of patients on discontinuation of medication, after overnight fasting, and after oral potassium administration. It has been suggested that the repolarisation effects may be 'benign' ${ }^{\prime 10}$ and that not all are associated with more serious cardiac consequences.'

\section{QT changes}

Normal corrected QT (QTc) values are not universally established because so many variables (gender, time of day, dief) affect 
measurement. However, a consensus appears to be emerging of a normal QTc upper limit of $450 \mathrm{~ms}$ for males and $470 \mathrm{~ms}$ for females, with a 'red zone' limit of 500 ms for both genders. A prolonged QT interval can initiate ectopic cardiac beats that may evolve into a potentially lethal ventricular tachyarrhythmia, called torsades de pointes, which is generally unresponsive to the usual anti-arrhythmic drugs. ${ }^{12-14}$

The risk of drug-induced torsades de pointes is increased under certain conditions, viz. structural heart disease, intracranial lesions, electrolyte abnormalities, hypothyroidism, pre-existing QT prolongation, QT dispersion, sinus bradycardia and polymorphic ventricular premature beats. ${ }^{15}$ It can also be induced by drugs, viz. antihistamines, antimalarials, antifungals, macrolide antibiotics, prokinetics and psychotropics. ${ }^{16,17}$ These effects may be indirect through cytochrome P450 enzymes (fluoxetine, fluvoxamine, and ketoconazole), or a direct effect (tricyclic antidepressants, antihistamines and anti-infectives). It is important to note that the correlation between prolonged QTc and torsades de pointes is not always a direct one in that there are a number of medications that prolong QTc but do not cause torsades de pointes.

The prevalence of torsades de pointes in the psychiatric population is unknown, but estimates from antiarrhythmic-induced torsades de pointes in the cardiac population range from 3\% to $15 \%$. Although usually self-limiting, torsades de pointes tends to recur, and in $31 \%$ of cases progresses to ventricular fibrillation and sudden death. ${ }^{18}$ Being associated with entirely nonspecific symptoms such as palpitations, dizziness, syncope and seizures, its potential seriousness may easily be misconstrued as primary psychiatric disorder, which can have a fatal outcome.

Antipsychotics differ in their capacity for QT prolongation." Among the antipsychotic drugs, the low-potency typical antipsychotics have most often been implicated. The highpotency typical and the atypical antipsychotics are less frequently associated with torsades de pointes; however they lespecially ziprasidone) have raised much debate and serious concern, which caused the Food and Drug Administration (FDA) to delay approval in some instances. Goodnick et al. ${ }^{19}$ report that the greatest concern is directed at the immediate use of haloperidol, the shortterm use of thioridazine, and the long-term use of clozapine and olanzapine.

Among the antidepressants, the tertiary tricyclic antidepressants (imipramine, amitriptyline) appear to have a more general impact, while the secondary tricyclic antidepressants (nortriptyline, desipramine) may impact more on children and the elderly. Among other antidepressants, the only reports of torsades de pointes appear to have occurred with mirtazapine. There are no effects on QTc by sertraline, citalopram, paroxetine and bupropion. Lithium and the benzodiazepines show little effect on the QTc, although there may be effects on other cardiovascular parameters. ${ }^{19}$

Additional risk factors for QT prolongation and torsades de pointes in the psychiatric population include deliberate or accidental antipsychotic overdose, co-morbid substance misuse and, in particular, the effects of high sympathetic arousal during restraint.

Because of a lack of resources in South Africa it is common for psychotropic medication to be initiated and maintained in an outpatient setting. At most, monitoring of these patients can only be done on a monthly basis, often by a psychiatric nurse. As this group of patients is at high risk for the cardiac side-effects of psychotropic medication and they are not well monitored by trained staff, there is a need to establish the safety of these drugs in our everyday clinical practice. The aim of this study was to determine the ECG changes in a group of outpatients on chronic psychotropic medication and the association, if any, with factors such as gender, age, co-morbid medical illness and concomitant medication.

\section{Subjects and methods}

\section{Subjects}

A cross-sectional study of all patients aged 18 years and older attending the outpatient departments of Chris Hani Baragwanath and Johannesburg hospitals was undertaken during the period September 2004 - November 2004. Patients were included if they were psychiatrically stable and had been on psychotropic medication for more than 6 months. Pregnant women were excluded from the study. The University of the Witwatersrand Human Research Ethics Committee approved the study.

\section{Procedures}

After obtaining written informed consent, the subjects' demographic data (age, race and gender) were recorded as well as presence of co-morbid medical illness and all medication currently used. The subjects then had a resting ECG recorded, which was analysed by the ECG machine and checked by a physician with respect to the rate, rhythm and other parameters.

\section{Statistical analysis}

The outcome variable was an abnormal ECG recording, and the factors considered were age, gender, race and the presence of comorbid illness. Descriptive statistics were computed as mean and 
frequencies (count and percentages). The two-sample ttest was used to compare the continuous characteristics (age) between the groups. Comparisons between the outcome variable with respect to the exposure variables were examined using contingency tables (chi-squared test with Yates's correction and Fisher's exact test). Logistical regression was computed to determine any significant associations between QTc and exposure variables. All analysis was done using Statistical Package for Social Sciences 10.0 for Windows (SPSS Inc., Chicago, III.). A value of $p<0.05$ was considered significant.

\section{Results}

About 150 patients attended the outpatient clinics during this period but only 80 patients volunteered to be included in the study. The mean age of the subjects was 45.4 (standard deviation (SD) 18.2) years, with a minimum age of 18 and a maximum of 86 years. Fifty-four (67.5\%) had evidence of some ECG abnormalities (Table I). There was no significant difference between ECG abnormalities and the different age groups $\left(\chi^{2}=\right.$ 3.77, $p>0.05)$, gender $\left(\chi^{2}=0.66, p>0.05\right)$, and the different race groups $\left(\chi^{2}=1.86 ; p>0.05\right)$.

Sixty-one patients (76.3\%) had no co-morbid medical illness and were on psychotropic medication only. Of these, 43 (70.5\%) had an abnormal ECG tracing compared with 18 (29.5\%) with normal tracings $\left(\chi^{2}=0.43, p>0.05\right)$.

The psychotropic medication that the patients were receiving included antipsychotics (haloperidol, trifluoperazine, risperidone, clozapine, olanzapine, quetiapine and sulpiride), antidepressants lamitriptyline, clomipramine, mianserin, fluoxetine, citalopram and venlafaxine), and mood stabilisers (lithium, valproate, carbamazepine and lamotrigine). Patients were either receiving antipsychotics alone or a combination of antipsychotic and/or antidepressant and/or mood stabiliser.

The ECG abnormalities recorded included prolonged or borderline QT interval (8.2\%), abnormal rate $(28.8 \%)$, irregular rhythm $(5.5 \%)$, prolonged PR interval $(2.7 \%)$, abnormal QRS complex (17.8\%), abnormal T wave (15.4\%), and abnormal ST segment (20.5\%) (Table II). The ECG abnormalities occurred more frequently in patients on antipsychotic medication alone or if it was combined with an anticonvulsant. The abnormalities were less frequent if the patient was on an antidepressant alone or in combination with an antipsychotic.

There was a significant positive correlation between the corrected QT interval and age $(r=0.43, p=0.0001)$ (Fig. la) and between corrected $Q T$ interval and female gender $(r=0.31, p=$ 0.006 ) (Fig. 1 b). There was no correlation between corrected QT interval and the treatment of a co-morbid illness $(r=-0.13, p>$ 0.051 .

\section{Discussion}

In this study the chronic use of common psychotropic medication was associated with abnormalities in the ECG tracings. Similar ECG changes such as rate, rhythm, T waves and QT interval changes have commonly been reported in other studies of patients

\section{Table I. Characteristics of the total patient sample (N (\%))}

\begin{tabular}{|c|c|c|c|}
\hline Variables & $\begin{array}{l}\text { Study population } \\
\qquad(N=80)\end{array}$ & $\begin{array}{l}\text { Abnormal ECG } \\
\qquad(N=54)\end{array}$ & $\begin{array}{c}\text { Normal ECG } \\
\qquad(N=26)\end{array}$ \\
\hline \multicolumn{4}{|c|}{ Age groups (years) } \\
\hline $18-30$ & $17(21.3)$ & $13(24.1)$ & $4(15.4)$ \\
\hline $31-45$ & $24(30)$ & $14(25.9)$ & $10(38.5)$ \\
\hline $46-60$ & $20(25)$ & $16(29.6)$ & $4(15.4)$ \\
\hline$>60$ & $19(23.7)$ & $11(20.4)$ & $8 \quad(30.8)$ \\
\hline \multicolumn{4}{|l|}{ Gender } \\
\hline Female & $44(55)$ & $28(51.9)$ & $16(61.5)$ \\
\hline Male & $36(45)$ & $26(48.1)$ & $10(38.5)$ \\
\hline \multicolumn{4}{|l|}{ Race } \\
\hline Black & $50(62.5)$ & $34(63)$ & $16(61.5)$ \\
\hline White & $30(37.5)$ & $20(37)$ & $10(38.5)$ \\
\hline \multicolumn{4}{|c|}{ Co-morbid medical illness } \\
\hline No & $61(76.3)$ & $43(79.6)$ & $18(69.2)$ \\
\hline Yes & $19(23.7)$ & $11(20.4)$ & $8(30.8)$ \\
\hline
\end{tabular}




\section{Table II. Frequency of the various types of ECG changes}

\begin{tabular}{|c|c|c|c|c|}
\hline Type of abnormality & $\begin{array}{c}\text { Total number (\%) } \\
\text { of ECG } \\
\text { abnormalities }\end{array}$ & $\begin{array}{c}\text { Number of ECG } \\
\text { abnormalities } \\
\text { with } \\
\text { antipsychotics } \\
\text { alone }\end{array}$ & $\begin{array}{c}\text { Number of ECG } \\
\text { abnormalities } \\
\text { with } \\
\text { combination } \\
\text { of antipsychotic } \\
\text { and antidepressant }\end{array}$ & $\begin{array}{c}\text { Number of ECG } \\
\text { abnormalities } \\
\text { with } \\
\text { combination } \\
\text { of antipsychotic } \\
\text { and } \\
\text { anticonvulsant }\end{array}$ \\
\hline QTC & $4(5.5)$ & 1 & 0 & 1 \\
\hline $\begin{array}{l}>440 \mathrm{~ms} \\
420-440 \mathrm{~ms}\end{array}$ & $2(2.7)$ & 0 & 0 & 0 \\
\hline \multicolumn{5}{|l|}{ Rate } \\
\hline$<60$ bpm & $8(11)$ & 1 & 0 & 3 \\
\hline$>80$ bpm & $13(17.8)$ & 3 & 1 & 5 \\
\hline Irregular rhythm & $4(5.5)$ & 0 & 0 & 2 \\
\hline Prolonged PR interval & $2(2.7)$ & 0 & 0 & 1 \\
\hline Abnormal QRS complex & $13(17.8)$ & 4 & 1 & 5 \\
\hline Abnormal T wave & $12(15.4)$ & 3 & 0 & 5 \\
\hline Abnormal ST segment & $15(20.5)$ & 3 & 2 & 5 \\
\hline
\end{tabular}

on psychotropic medication. ${ }^{20-22}$ However, most of the changes recorded are considered 'benign' ${ }^{23}$ and may also be seen in

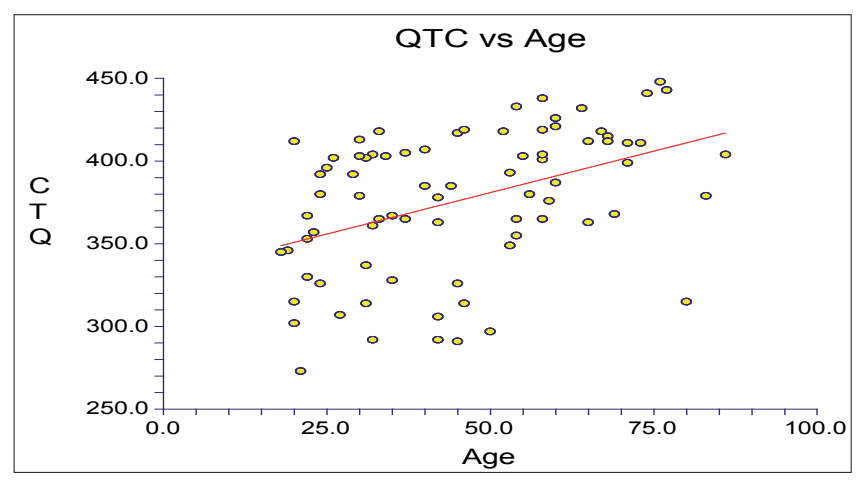

Fig. 1a. Correlation between corrected QT interval and age.

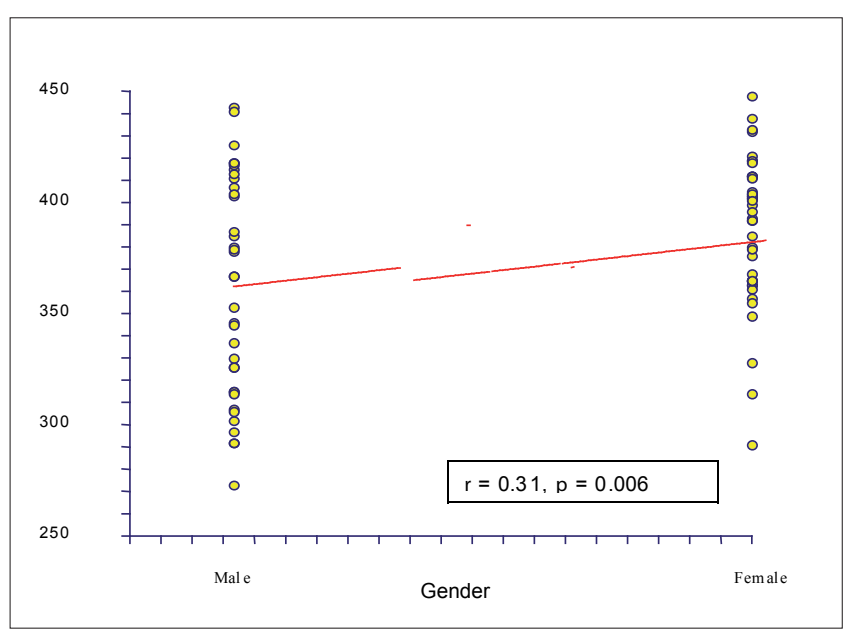

Fig. 1b. Correlation between corrected QT interval and gender. athletes without demonstrable organic heart disease, chronic schizophrenics not receiving any psychotropic medication, physically healthy persons under certain stressful conditions, ${ }^{10}$ and patients on placebo therapy. ${ }^{24}$

Unlike other studies, ${ }^{25}$ frequency of ECG changes did not increase with age or concomitant use of other medication. Concern about prescribing psychotropic medication is greatest in the case of the elderly and patients with co-morbid medical illness. Patients with co-morbid illnesses are more susceptible to the side-effects of psychotropics because of disturbed drug distribution and metabolism and because of the likelihood of interactions between psychotropic and non-psychotropic medication. It is possible that some of the patients exhibiting ECG abnormalities may have some as yet undiagnosed and untreated cardiac pathology.

Previous studies have shown that predictors of QTc lengthening include age over 65 years, use of tricyclic antidepressants and antipsychotics, antipsychotic dose, female gender, bradycardia, electrolyte imbalances, cardiac diseases, simultaneous use of multiple drugs prolonging QT interval, and genetic predisposition. This study confirmed a significant positive correlation between the corrected QT interval and age and female gender but did not show any correlation with a bradycardia, simultaneous use of multiple drugs or pre-existing medical illness. It is likely that this is because of the small sample size rather than any fundamental difference in characteristics of this study population.

This study is limited in its generalisability because all subjects were attendees of a tertiary hospital outpatient department. The small sample size may have statistical limitations in ascertaining meaningful differences when comparing groups. Finally, the 
cross-sectional nature of the sample did not allow us to establish a cause-and-effect relationship as there was no baseline ECG recording before the commencement of psychotropics, no comparator groups and no multiple prospective ECG recordings. Notwithstanding these limitations the changes in ECG recordings of patients in this study were significant.

\section{Conclusion}

Psychotropic drugs have properties that result in ECG changes in ordinary doses and there is much concern about these cardiac effects and their relation to sudden death. This study serves to provide some evidence to mental health care practitioners in limited-resources settings that it is relatively safe to initiate and titrate psychotropic medication in an outpatient setting. However, it would be prudent to ask apparently healthy patients if they have had syncope, if they have relatives with long QT syndrome, or if they have relatives who died suddenly at a young age, before initiating treatment with psychotropic medication. Among older patients, especially those with known heart disease or taking drugs that can prolong QT, a pre-treatment ECG would be appropriate.

Finally, before prescribing a medicinal product that prolongs QT interval, physicians should carefully evaluate not only the disease they want to treat but also the availability of equally effective, alternative drugs. One of the most basic ethical principles of medicine requires that the beneficial effects expected from a therapy should, for each treated patient, outweigh any possible adverse consequence, particularly when the latter could be lethal.

The Division of Psychiatry, University of the Witwatersrand, assisted in the funding of this study.

The authors would like to acknowledge Dr A Kalaba from Chris Hani Baragwanath Hospital for her assistance in gathering the data.

\section{References}

1. Waddington JL, Youssef HA, Kinsella A. Mortality in schizophrenia - antipsychotic polypharmacy and absence of adjunctive anticholinergics over the course of a 10-year prospective study. Br J Psychiatry 1998; 173: $325-329$
2. Ruschena $D$, Mullen PE, Burgess $P$, et al. Sudden death in psychiatric patients. BrJ Psychiatry 1998; 172: 331-336.

3. Politi P, Piccinelli M, Klersy C, et al. Mortality in psychiatric patients 5 to 21 years after hospital admission in Italy. Psychol Med 2002; 32: 227-237

4. Hannerz H, Borga P. Mortality among persons with a history as psychiatric inpatients with functional psychosis. Soc Psychiatry Psychiatr Epidemio 2000: 35: 380-387

5. Kilian JG, Kerr K, Lawrence $C$, et al. Myocarditis and cardiomyopathy associated with clozapine. Lancet 1999; 354: 1841-1845.

6. Agelink MW, Majewski T, Wurthmann C, et al. Effects of newer atypical antipsychotics on autonomic neurocardiac function: a comparison between amisulpride, olanzapine, sertindole, and clozapine. I Clin Psychopharmacol $2001 ; \mathbf{2} 1: 8-3$.

7. Schwalb $H$, Eckmann $F$, van Eimeren W. ECG changes in psychiatric patients under long-term therapy with psychopharmacology. Fortsch Neurol Psychiatr 1978; 46: 484-490

8. Burrows GD, Vohra J, Hunt D, et al. Cardiac effects of different tricyclic antidepressant drugs. Br J Psychiatry 1976; 129: 335-34 1.

9. Roose SP, Glassman AH, Giardina EGV, et al. Tricyclic antidepressants in depressed patients with cardiac conduction disease. Arch Gen Psychiatry 1987; 44: 273-275.

10. Wendkos $M H$. Cardiac changes related to phenothiazine, with special reference to thioridazine. J Am Geriatr Soc 1967; 15: 20-28.

11. Reilly JG, Ayis SA, Jones SJ, et al. QTc-interval abnormalities and psychotropic drug therapy in psychiatric patients. Lancet 2000; 355: 1048-1052.

12. Calderone V, Cavero I. Ventricular arrhythmias. A potential risk associated with the use of non-cardiovascular drugs prolonging the QT interval. Minerva Med 2002; 93: 181-197.

13. Woosley RL, Chen Y, Freiman JP, et al. Mechanism of the cardiotoxic actions of terfenadine. JAMA 1993; 269: 1532-1536.

14. Dessertenne F. Ventricular tachycardia with two variable opposing foci. Arch Mal Coeur Vaiss 1996; 59: 263-272

15. Hatta K, Takahashi T, Nakamura H, et al. Hypokalemia and agitation in acute psychotic patients. Psychiatry Res 1999; 86: 85-88.

16. Yap YG, Camm J. Risk of torsades de pointes with non-cardiac drugs. BM 2000; 320: $1158-1159$

17. Roden DM. Torsades de pointes. Clin Cardiol 1993; 16: 683-686

18. Bednar MM, Harrigan EP, Anziano RJ, et al. The QT interval. Prog Cardiovasc Dis 2001; 43: 1-45

19. Goodnick PJ, Jerry J, Parra F. Psychotropic drugs and the ECG: focus on the QTc interval. Expert Opin Pharmacother 2002; 3: 479-498.

20. Grauaner KF, Murphree DD. Electrocardiographic changes associated with thioridazine. J Neuropsychiat 1996; 5: 344.

21. Dillenkoffer RL, Gallant DM, Phillips JH. Electrocardiographic evaluation of mesoridazine. Curr Ther Res 1974; 14: 71

22. Brannan MD, Riggs JJ, Hageman WE, et al. A comparison of the cardiovascular effects of haloperidol, thioridazine and chlorpromazine $\mathrm{HCl}$. Arch Int J Pharmacodyn 1980; 244: 48 .

23. Elert MH, Shader RI. Cardiovascular effects of psychotropic drugs. Conn Med 1969; 33: 695.

24. Holden M, Itil TM. Electrocardiographic changes with psychotropic drugs. In: Wheatley D, ed. Stress and the Heart. New York: Raven Press, 1997 87

25. Eckmann F, Schwalb H, Hanika R. Psychotropic agents and pathological ECG changes. Pharmakopsychiatr Neuropsychopharmakol 1975; 8: 57-68. 